\title{
OSCILLATION THEOREMS FOR A SECOND ORDER SUBLINEAR ORDINARY DIFFERENTIAL EQUATION
}

\author{
TAKESHI KURA
}

\begin{abstract}
New oscillation criteria are given for the differential equation
\end{abstract}

$$
u^{\prime \prime}+a(t)|u|^{\alpha} \operatorname{sgn} u=0, \quad 0<\alpha<1,
$$

where $a(t)$ is allowed to take on negative values for arbitrarily large $t$.

We consider the sublinear differential equation

$$
u^{\prime \prime}+a(t)|u|^{\alpha} \operatorname{sgn} u=0, \quad 0<\alpha<1,
$$

where $a(t)$ is continuous on $\left[t_{0}, \infty\right), t_{0}>0$. We restrict our attention to proper solutions of (1), that is, those solutions $u(t)$ which exist on some ray $\left[T_{u}, \infty\right) \subset\left[t_{0}, \infty\right)$ and satisfy $\sup \{|u(t)|: t \geqslant T\}>0$ for any $T \geqslant T_{u}$. A proper solution is called oscillatory if it has arbitrary large zeros; otherwise it is called nonoscillatory. Equation (1) is said to be oscillatory if all of its proper solutions are oscillatory.

Belohorec [1] has shown that, in case $a(t)$ is nonnegative, equation (1) is oscillatory if and only if

$$
\int_{t_{0}}^{\infty} t^{\alpha} a(t) d t=\infty
$$

Of particular interest, therefore, is the problem of finding criteria for the oscillation of equation (1) when $a(t)$ is allowed to take on negative values for arbitrarily large $t$. This problem has been studied by various authors including Belohorec [2], Butler [3, 4], Grammatikopoulos [5], Kamenev [6] and Wong [7]. We refer in particular to the following oscillation criteria for (1) due, respectively, to Belohorec [2] and Kamenev [6]:

$$
\begin{gathered}
\int_{t_{0}}^{\infty} t^{\beta} a(t) d t=\infty \quad \text { for some } \beta \in[0, \alpha] \\
\limsup _{t \rightarrow \infty} \frac{1}{t} \int_{t_{0}}^{t} \int_{t_{0}}^{s} a(\tau) d \tau d s=\infty
\end{gathered}
$$

The purpose of this paper is to proceed further in this direction to present new oscillation theorems which unify and considerably improve the above-mentioned results of Belohorec and Kamenev.

Received by the editors May 8, 1981.

1980 Mathematics Subject Classification. Primary 34C10.

Key words and phrases. Oscillation, oscillatory solution, nonlinear, sublinear differential equation. 
Our main results are as follows:

THEOREM 1. Equation (1) is oscillatory if

$$
\underset{t \rightarrow \infty}{\limsup } \frac{1}{t} \int_{t_{0}}^{t} \int_{t_{0}}^{s} \tau^{\beta} a(\tau) d \tau d s=\infty \quad \text { for some } \beta \in[0, \alpha]
$$

THEOREM 2. Let $\beta \in[0, \alpha)$. Equation (1) is oscillatory if there exists a continuous function $f(t)$ on $\left[t_{0}, \infty\right)$ such that

$$
\underset{T \rightarrow \infty}{\limsup } \frac{1}{T} \int_{t}^{T} \int_{t}^{s} \tau^{\beta} a(\tau) d \tau d s \geqslant f(t)
$$

for $t \in\left[t_{0}, \infty\right)$ and

$$
\int_{t_{0}}^{\infty} \frac{f_{+}^{2}(t)}{t} d t=\infty
$$

where $f_{+}(t)=\max \{f(t), 0\}$.

Proof of Theorems 1 AND 2. Suppose to the contrary that there exists a nonoscillatory solution $u(t)$ of (1). Without loss of generality we may suppose that $u(t)>0$ on $\left[t_{1}, \infty\right), t_{1}>t_{0}$. Put

$$
v(t)=\frac{1}{1-\alpha} t^{\beta} u^{1-\alpha}(t)
$$

It is easy to verify that

$$
v^{\prime \prime}(t)=-\frac{\alpha}{1-\alpha} \frac{1}{v(t)}\left(v^{\prime}(t)-\frac{\beta v(t)}{\alpha t}\right)^{2}-\frac{\beta(\alpha-\beta)}{\alpha} \frac{v(t)}{t^{2}}-t^{\beta} a(t) .
$$

Integrating (8) twice over $[t, T], t \geqslant t_{1}$, we have

$$
\begin{gathered}
v(t)-v(T)+v^{\prime}(t)(T-t)=\frac{\alpha}{1-\alpha} \int_{t}^{T} \int_{t}^{s} \frac{1}{v(\tau)}\left(v^{\prime}(\tau)-\frac{\beta v(\tau)}{\alpha \tau}\right)^{2} d \tau d s \\
+\frac{\beta(\alpha-\beta)}{\alpha} \int_{t}^{T} \int_{t}^{s} \frac{v(\tau)}{\tau^{2}} d \tau d s+\int_{t}^{T} \int_{t}^{s} \tau^{\beta} a(\tau) d \tau d s .
\end{gathered}
$$

Suppose (5) holds for some $\beta \in[0, \alpha]$. Divide (9) by $T$ and take the upper limit as $T \rightarrow \infty$. Using (5), we then see that

$$
v^{\prime}(t) \geqslant \limsup _{T \rightarrow \infty} \frac{1}{T} \int_{t}^{T} \int_{t}^{s} \tau^{\beta} a(\tau) d \tau d s=\infty
$$

for all $t \geqslant t_{1}$. This contradiction proves Theorem 1 .

Let $\beta \in[0, \alpha)$ and suppose there is a function $f(t)$ satisfying (6) and (7). Dividing (9) by $T$, taking the upper limit as $T \rightarrow \infty$ and using (6), we obtain

$$
\begin{aligned}
\liminf _{T \rightarrow \infty} \frac{v(T)}{T} & +\frac{\alpha}{1-\alpha} \int_{t}^{\infty} \frac{1}{v(s)}\left(v^{\prime}(s)-\frac{\beta v(s)}{\alpha s}\right)^{2} d s \\
& +\frac{\beta(\alpha-\beta)}{\alpha} \int_{t}^{\infty} \frac{v(s)}{s^{2}} d s+f(t) \leqslant v^{\prime}(t), \quad t \geqslant t_{1},
\end{aligned}
$$


which shows that

$$
\begin{aligned}
\liminf _{T \rightarrow \infty} \frac{v(T)}{T}<\infty, & \int_{t}^{\infty} \frac{1}{v(s)}\left(v^{\prime}(s)-\frac{\beta v(s)}{\alpha s}\right)^{2} d s<\infty \\
& \int_{t}^{\infty} \frac{v(s)}{s^{2}} d s<\infty
\end{aligned}
$$

for $t \geqslant t_{1}$. On the other hand, integrating the equality

$$
\frac{2 \beta}{\alpha}\left(\frac{v(t)}{t}\right)^{\prime}+\frac{1}{v(t)}\left(v^{\prime}(t)-\frac{\beta v(t)}{\alpha t}\right)^{2}=\frac{v^{\prime 2}(t)}{v(t)}+\frac{\beta^{2}-2 \alpha \beta}{\alpha^{2}} \frac{v(t)}{t^{2}}
$$

we have

$$
\begin{aligned}
\frac{2 \beta}{\alpha}\left(\frac{v(T)}{T}-\frac{v(t)}{t}\right) & +\int_{t}^{T} \frac{1}{v(s)}\left(v^{\prime}(s)-\frac{\beta v(s)}{\alpha s}\right)^{2} d s \\
& =\int_{t}^{T} \frac{v^{\prime 2}(s)}{v(s)} d s+\frac{\beta^{2}-2 \alpha \beta}{\alpha^{2}} \int_{t}^{T} \frac{v(s)}{s^{2}} d s .
\end{aligned}
$$

Taking the lower limit as $T \rightarrow \infty$ in (12), we conclude with the aid of (11) that

$$
\int_{t}^{\infty} \frac{v^{\prime 2}(s)}{v(s)} d s<\infty, \quad t \geqslant t_{1} .
$$

By Schwarz's inequality we have.

$$
t \int_{t_{1}}^{\infty} \frac{v^{\prime 2}(s)}{v(s)} d s \geqslant\left(\int_{t_{1}}^{t} \frac{v^{\prime}(s)}{v^{1 / 2}(s)} d s\right)^{2}=4\left(v^{1 / 2}(t)-v^{1 / 2}\left(t_{1}\right)\right)^{2},
$$

which, together with (13), implies that

$$
v(t) \leqslant k t, \quad t \geqslant t_{2},
$$

for some constants $k>0$ and $t_{2}>t_{1}$. Since $\max \left\{v^{\prime}(t), 0\right\} \geqslant f_{+}(t) \geqslant 0$ by (10), it follows from (13) and (14) that

$$
\frac{1}{k} \int_{t_{2}}^{\infty} \frac{f_{+}^{2}(t)}{t} d t \leqslant \int_{t_{2}}^{\infty} \frac{v^{\prime 2}(t)}{v(t)} d t<\infty
$$

which contradicts (7). This completes the proof of Theorem 2.

EXAMPLE 1. Consider the equation

$$
u^{\prime \prime}+\left(t^{\gamma} \sin t\right)|u|^{\alpha} \operatorname{sgn} u=0, \quad 0<\alpha<1,
$$

for $t>0$, where $\gamma$ is a constant. If $\beta+\gamma>1$, then

$$
\underset{T \rightarrow \infty}{\lim \sup } \frac{1}{T} \int_{t}^{T} \int_{t}^{s} \tau^{\beta+\gamma} \sin \tau d \tau d s=\infty,
$$

so that, by Theorem 1 , (15) is oscillatory when $\gamma>1-\alpha$. If $0<\beta+\gamma \leqslant 1$, then we have

$$
\begin{aligned}
\underset{T \rightarrow \infty}{\limsup } \frac{1}{T} \int_{t}^{T} \int_{t}^{s} \tau^{\beta+\gamma} \sin \tau d \tau d s \geqslant t^{\delta} \cos t-\delta t^{\delta-1} \sin t \\
\\
\quad-\delta(\delta-1) t^{\delta-2} \cos t-\delta(\delta-1)(\delta-2) \int_{t}^{\infty} s^{\delta-3} \cos s d s
\end{aligned}
$$


where $\delta=\beta+\gamma$. Put $f(t)=t^{\delta} \cos t-2 \delta$. Then (6) holds for $t \geqslant t_{1}$, provided $t_{1}$ is sufficiently large. Since $\delta>0$, there is an integer $N>0$ such that $2 N \pi-(\pi / 4)>t_{1}$ and

$$
f(t) \geqslant 2^{-1 / 2} \text { on }[2 n \pi-\pi / 4,2 n \pi+\pi / 4],
$$

for all $n \geqslant N$. It follows that

$$
\int_{t_{1}}^{\infty} \frac{f_{+}^{2}(t)}{t} d t \geqslant \sum_{n=N}^{\infty} \int_{2 n \pi-(\pi / 4)}^{2 n \pi+(\pi / 4)} \frac{d t}{2 t}=\frac{1}{2} \sum_{n=N}^{\infty} \log \left(1+\frac{2}{8 n-1}\right)=\infty,
$$

and so (7) is satisfied. Applying Theorem 2, we see that (15) is oscillatory if $-\alpha<\gamma \leqslant 1-\alpha$. We conclude therefore that equation (15) is oscillatory if $\gamma>-\alpha$. (Butler [4] conjectures that (15) is oscillatory if and only if $\gamma \geqslant-\alpha$.)

We note that Belohorec's criterion (3) does not apply to (15) and that Kamenev's criterion (4) assures the oscillation of (15) only for $\gamma>1$.

REMARK. In Theorem 2 the assumption that $\beta<\alpha$ is essential. In fact, consider the equation (1) in which $a(t)=t^{-\alpha-1}(\log t)^{-3 / 2}$ for $t>1$. As easily verified,

$$
\underset{T \rightarrow \infty}{\limsup } \frac{1}{T} \int_{t}^{T} \int_{t}^{s} \tau^{\alpha} a(\tau) d \tau d s=2(\log t)^{-1 / 2}, \quad t>1,
$$

so that conditions (6) and (7) are satisfied with $\beta=\alpha$ and $f(t)=2(\log t)^{-1 / 2}$. However, since $a(t)>0$ and $\int^{\infty} t^{\alpha} a(t) d t<\infty$, the equation in question is not oscillatory according to Belohorec's theorem [1].

ACKNOWLEDGement. The author would like to thank Professor Takaŝi Kusano and the referee for their helpful comments and suggestions.

\section{REFERENCES}

1. S. Belohorec, Oscillatory solutions of certain nonlinear differential equations of the second order, Mat.-Fyz. Časopis Sloven. Akad. Vied. 11 (1961), 250-255.

2. _ Two remarks on the properties of solutions of a nonlinear differential equation, Acta Fac. Rerum Natur. Univ. Comenian. Math. 22 (1969), 19-26.

3. G. J. Butler, Oscillation theorems for a nonlinear analogue of Hill's equation, Quart. J. Math. Oxford Ser. (2) 27 (1976), 159-171.

4. Integral averages and the oscillation of second order ordinary differential equations, SIAM J. Math. Anal. 11 (1980), 190-200.

5. M. K. Grammatikopoulos, Oscillation theorems for second order ordinary differential inequalities and equations with alternating coefficients, An. Stiint. Univ. “Al. I. Cuza” Iasi Sect. I a Mat. 26 (1980), 67-76.

6. I. V. Kamenev, Some specifically nonlinear oscillation theorems, Mat. Zametki 10 (1971), 129-134. (Russian)

7. J. S. W. Wong, Oscillation theorems for second order nonlinear differential equations, Bull. Inst. Math. Acad. Sinica 3 (1975), 283-309.

Department of Mathematics, Faculty of Science, Hiroshima University, Hiroshima 730, JAPAN 